\title{
Basic Study for Quantification of Movement Disorders in Parkinson's Patients Using IMU Sensor
}

\author{
D. J. Park ${ }^{1}$, J. W. Lee' ${ }^{1}$ W. B. Jeong ${ }^{1}$, S. J. Ahn ${ }^{2}$ \\ ${ }^{1}$ School of Mechaninal Engineering, Pusan National University \\ Jangjoen-dong, Kumjung-ku, 609-735, Busan, Korea \\ ehdwns5142@naver.com; qwer5209@pusan.ac.kr; wbjeong@pusan.ac.kr \\ ${ }^{2}$ Division of Energy and Electric Engineering, Uiduk University \\ 780-713, Gyeongju, Korea \\ sjahn@uu.ac.kr
}

\section{Extended Abstract}

In the international community, population aging is leading to an increase in the number of Parkinson's patients. Therefore, there is a need for effective diagnosis of Parkinson's disease. At present, when diagnosing Parkinson's disease, the medical doctor asks the Parkinson's patient for a specific movement and visually identifies the movement. The medical doctor diagnoses qualitatively by subjective and empirical judgment. However, the diagnostic results can be not consistently varied by the medical doctor's individual judgment. In addition, it is difficult to effectively manage progress and change in disease degree while the patient is treated by any medicine or remedy. Therefore, a quantitative diagnostic method that can objectively diagnose and effectively manage movement disorders in Parkinson's patients is needed. In this study, we aimed to develop methods to quantify movement disorders in Parkinson's patients using IMU acceleration sensor. The first method of this study is to quantitatively measure the specific movement of Parkinson's patients by motion tracking. ${ }^{[1,2,3]}$ In this method, a quantitative value is calculated by applying a frequency filter that can effectively extract motion characteristics to an acceleration signal measuring a specific movement of Parkinson's patients using the IMU sensor. It can calculate the amount of motion of the specific movement in three-dimensional space by performing coordinate transformation and numerical integration using frequency-filtered Euler angles and quaternions representing the rotation and direction of the movement. This method requires an algorithm that minimizes the draft error generated in the process of calculating the displacement by numerical integration of the acceleration signal. In this study, it is shown that accumulation of numerical integration error can be improved by using the Kalman filter theory and the reference point of repeated movement. The second method of this study is to extract the feature that can represent the motion characteristic from the measured acceleration signal for the specific movement and use it as a quantitative index. ${ }^{[4,5]}$ Feature values of Skewness, Kurtosis, Cepstrum, and Envelope detection calculated for the acceleration signal applied to the frequency filter which can effectively extract motion characteristics can be quantification indices. When we analyzed the IMU acceleration signal for normal movement and for movement assumed Parkinson's patient as an preliminary study, we found that there is a clear difference in specific frequencies associated with movement. In future studies, standardized movements to classify Parkinson's patients in cooperation with hospital medical doctors will be established. And the various quantitative index values calculated by signal processing of the acceleration measured by the IMU sensor will be correlated with the degree of disease diagnosed by medical doctors. We will apply various statistical methods including nonparametric statistical methods to derive reliable correlations. $^{[6]}$

\section{Acknowledgement}

This research was supported by Basic Science Research Program through the National Research Foundation of Korea(NRF) funded by the Ministry of Education(NRF-2017R1D1A1B03028674).

\section{References}

[1] J. Yong An, Y. Gwon Kim and S. Teak Chung, "A Research on Measurements and Contents of the Cervical Range of Motion Based on Inertial Measurement Unit,” Korean Society For Computer Game, vol. 28, no. 3, pp. 143-150, 2015. 
[2] P. Daponte, L. De Vito, M. Riccio and C. Sementa, "Design and validation of a motion-tracking system for ROM measurements in home rehabilitation," Measurement, vol. 55, pp. 82-96, 2014.

[3] W. Yang, A. Bajenov, Y. Shen, "Improving low-cost inertial-measurement-unit (IMU)-based motion tracking accuracy for a biomorphic hyper-redundant snake robot," Robotics and biomimetics, vol. 4, no. 1, p. 16, 2017.

[4] U. Grüner, C. Eggers, M. Ameli, A. S. Sarfeld, G. R. Fink, and D. A. Nowak, "1 Hz rTMS preconditioned by tDCS over the primary motor cortex in Parkinson's disease: effects on bradykinesia of arm and hand," Journal of neural transmission, vol. 117, no. 2, pp. 207-216, 2010.

[5] S. K. Strambi, B. Rossi, G. De Michele, and S. Sello, "Effect of medication in Parkinson's disease: a wavelet analysis of EMG signals," Medical Engineering and Physics, vol. 26, no. 4, pp. 279-290, 2004.

[6] G. A. Gescheider, Psychophysics: the fundamentals. Psychology Press, 2013. 